\title{
ON THE EXISTENCE OF BOUNDED SOLUTIONS OF NONLINEAR ELLIPTIC SYSTEMS
}

\author{
ABDELAZIZ AHAMMOU \\ Received 24 March 2000 and in revised form 13 August 2000
}

\begin{abstract}
We study the existence of bounded solutions to the elliptic system $-\Delta_{p} u=f(u, v)+h_{1}$ in $\Omega,-\Delta_{q} v=g(u, v)+h_{2}$ in $\Omega, u=v=0$ on $\partial \Omega$, non-necessarily potential systems. The method used is a shooting technique. We are concerned with the existence of a negative subsolution and a nonnegative supersolution in the sense of Hernandez; then we construct some compact operator $T$ and some invariant set $K$ where we can use the Leray Schauder's theorem.
\end{abstract}

2000 Mathematics Subject Classification: 35J25, 35J60.

1. Introduction. The aim of this paper is to study the existence of solutions for the following system:

$$
\begin{gathered}
-\Delta_{p} u=f(u, v)+h_{1}, \quad-\Delta_{q} v=g(u, v)+h_{2} \quad \text { in } \Omega, \\
u=v=0 \quad \text { on } \partial \Omega,
\end{gathered}
$$

where $\Omega$ is a smooth bounded domain of $\mathbb{R}^{N}$, where $N \geq 1, p, q>1, f, g$ are continuous functions of $\mathbb{R}^{2}$ into $\mathbb{R}$ and $h_{1}, h_{2}$ are the functions given in $L^{+\infty}(\Omega)$.

System (1.1) results from the study of the nonlinear phenomena, such as the evolution of population, of chemical reaction, and so forth. A great attention was given to the existence of the solutions for a system of the (1.1) type, by using various approaches (cf. [3, 4, 5, 7, 13]). When the system has a variational structure, the existence of the solutions for (1.1) can be established by means of the variational approaches under adapted conditions (cf. [9, 13]). When (1.1) does not have a variational structure, as in Vélin and de Thélin [13], where the authors obtained some results for the existence of solutions to problem (1.1) with the following growth conditions of nonlinearity $f$ and $g$ :

$$
\begin{aligned}
& |f(u, v)| \leq a_{1}|u|^{\alpha_{0}}|v|^{\beta_{0}+1}+a_{2}|u|^{\alpha_{1}-1}+a_{3}|v|^{\beta_{1}-1}, \\
& |g(u, v)| \leq a_{4}|u|^{\alpha_{0}+1}|v|^{\beta_{0}}+a_{5}|u|^{\alpha_{2}-1}+a_{6}|v|^{\beta_{2}-1},
\end{aligned}
$$

where $a_{i}(i=1, \ldots, 6)$ are positive constants and $\alpha_{i}$ and $\beta_{i}(i=0,1,2)$ satisfy

$$
\begin{gathered}
\frac{\alpha_{0}+1}{p}+\frac{\beta_{0}+1}{q}<1, \\
1<\alpha_{1}<p ; \quad 0<\beta_{1}-1<\frac{q}{p^{*}}, \\
1<\alpha_{2}-1<\frac{p}{q^{*}} ; \quad 0<\beta_{2}<q .
\end{gathered}
$$


Always in the case of a system, we can notice the existence results obtained in Baoyao [2], and Brézis and Lieb [4].

The case of a scalar equation has been studied by many authors, see de Figueiredo and Gossez [6], Fernandes et al. [10] and Fonda et al. [11]. More recently, some interesting results have been obtained by Gossez and El Hachimi [12] and Anane and Chakrone [1]. Those authors derived the solvability of the following problem:

$$
\begin{gathered}
-\Delta_{m} u=f(u)+h \quad \text { in } \Omega, \\
u=0 \quad \text { on } \partial \Omega,
\end{gathered}
$$

under the following condition:

$$
\liminf _{u \rightarrow \infty} \frac{p F(u)}{u^{m}}<\mu_{m}^{\prime}
$$

where

$$
\mu_{m}^{\prime}=(m-1)\left[\frac{2}{R(\Omega)} \int_{0}^{1} \frac{d s}{\sqrt[m]{1-s^{m}}}\right]^{m},
$$

and $R(\Omega)$ denotes the radius of the smallest open ball $B(0, R)$ containing $\Omega$. The particular cases $N=1$ and $m=2$ were considered in [10]. It was shown there that (1.4) is solvable for any $h \in L^{\infty}(\Omega)$ if

$$
\lim _{u \rightarrow \infty} \inf \frac{2 F(u)}{u^{2}}<\lambda_{1,2},
$$

where $\lambda_{1,2}$ is the first eigenvalue of $-\Delta$ and $\left.\Omega=\right] a, b$. Observe that for $N>1$, we have $\mu_{2}^{\prime}<\lambda_{1,2}(\Omega)$. Then, the question naturally arises whether $\mu_{m}^{\prime}$ can be replaced by $\lambda_{1, m}(\Omega)$ in (1.5), where $\lambda_{1, m}$ is the first eigenvalue of $-\Delta_{m}$. This problem remains open.

The goal of this paper is to show that the same approach in [12] can be applied for some quasilinear elliptic systems with the constants $\mu_{p}$ and $\mu_{q}$, defined below, associated, respectively, with the operators $-\Delta_{p}$ and $-\Delta_{q}$, and where $\mu_{m}(m=p, q)$, better than $\mu_{m}^{\prime}$, is presented in (1.5). In this case, we treat the question of the existence of the solutions for system (1.1) without imposing variational structures, which is often the case for system (1.1) and without necessarily the growth conditions for $f$ and $g$.

2. Main result. We make the following assumptions:

$\left(\mathrm{H}_{1}\right)$ (i) The function $f(u, \cdot)$ is a nonincreasing function on $\mathbb{R}$ for all $u$ in $\mathbb{R}$,

(ii) The function $g(\cdot, v)$ is a nonincreasing function on $\mathbb{R}$ for all $v$ in $\mathbb{R}$.

$\left(\mathrm{H}_{2}\right)$ There exists some unbounded increasing subsequence $\left(m_{k}\right)_{k}$, satisfying

$$
\begin{aligned}
\lim _{k \rightarrow+\infty} \frac{p F\left(m_{k}^{1 / p}, m_{k}^{1 / q}\right)}{m_{k}}<\mu_{p}, & \lim _{k \rightarrow+\infty} \frac{q G\left(m_{k}^{1 / p}, m_{k}^{1 / q}\right)}{m_{k}}<\mu_{q}, \\
\lim _{k \rightarrow+\infty} \frac{p F\left(-m_{k}^{1 / p},-m_{k}^{1 / q}\right)}{m_{k}}<\mu_{p}, & \lim _{k \rightarrow+\infty} \frac{q G\left(-m_{k}^{1 / p},-m_{k}^{1 / q}\right)}{m_{k}}<\mu_{q},
\end{aligned}
$$


where $F$ and $G$ are the following functions:

$$
F(u, v)=\int_{0}^{u} f(s, v) d s, \quad G(u, v)=\int_{0}^{v} g(u, t) d t
$$

and where we denote by $\mu_{p}$ and $\mu_{q}$ the following constants:

$$
\mu_{p}=(p-1)\left[\frac{2}{b-a} \int_{0}^{1} \frac{d s}{\sqrt[p]{1-s^{p}}}\right]^{p}, \quad \mu_{q}=(q-1)\left[\frac{2}{b-a} \int_{0}^{1} \frac{d t}{\sqrt[q]{1-t^{q}}}\right]^{q}
$$

with $b-a=\min \left(b_{i}-a_{i}\right)$ and $P=\Pi\left[a_{i}, b_{i}\right]$ is the smallest cube such that $P \supset \Omega$.

Observe that for $N=1, \mu_{p}$ and $\mu_{q}$ are, respectively, the first eigenvalue of $-\Delta_{p}$ and $-\Delta_{q}$ when $\left.\Omega=\right] a, b\left[\right.$. It is clear that $\mu_{p}$ is better than $\mu_{p}^{\prime}$ defined in (1.5). In particular, it is interesting when $\Omega$ is a rectangle or a triangle, because $\mu_{p} \gg \mu_{p}^{\prime}$ and $\mu_{p} \approx \lambda_{1, p}(\Omega)$.

The main result of this paper is the following statement.

THEOREM 2.1. Under hypotheses $\left(H_{1}\right)$ and $\left(H_{2}\right)$. Problem (1.1) has a solution $(u, v)$ in $\left(W_{0}^{1, p}(\Omega) \times W_{0}^{1, q}(\Omega)\right) \cap\left(L^{+\infty}(\Omega) \times L^{+\infty}(\Omega)\right)$ for any $\left(h_{1}, h_{2}\right)$ in $L^{+\infty}(\Omega) \times L^{+\infty}(\Omega)$.

EXAMPLe 2.2. Consider

$$
f(u, v)=a(x) u|u|^{\alpha-1}|v|^{\beta+1}, \quad g(u, v)=b(x)|u|^{\gamma+1} v|v|^{\delta-1} .
$$

(1) Assume that $\|a\|_{\infty}<\mu_{p}$, $\|b\|_{\infty}<\mu_{q}$, and

$$
\frac{\alpha+1}{p}+\frac{\beta+1}{q} \leq 1, \quad \frac{\gamma+1}{p}+\frac{\delta+1}{q} \leq 1
$$

Then we conclude the existence of solutions.

(2) If

$$
\frac{\alpha+1}{p}+\frac{\beta+1}{q}<1, \quad \frac{\gamma+1}{p}+\frac{\delta+1}{q}<1,
$$

we have the existence for all $(a, b)$ in $L^{+\infty}(\Omega)$.

The method used in this paper is a shooting technique. In Section 3, we are concerned with the existence of a negative subsolution $\left(u_{0}, v_{0}\right)$ and a nonnegative supersolution $\left(u^{0}, v^{0}\right)$ in the sense of Hernandez's definition [13]. In Section 4, we consider some compact operator $T$ and some invariant set $K$. And, we look for solutions of problem (1.1) as fixed points of the operator $T$. We will be in the conditions of the Schauder fixed point theorem.

\section{Construction of sub-supersolutions}

Definition 3.1. A pair $\left[\left(u_{0}, v_{0}\right),\left(u^{0}, v^{0}\right)\right]$ is a weak sub-supersolution for the Dirichlet problem (1.1), if the following conditions are satisfied: 


$$
\begin{gathered}
\left(u_{0}, v_{0}\right) \in\left(W^{1, p}(\Omega) \times W^{1, q}(\Omega)\right) \cap\left(L^{+\infty}(\Omega) \times L^{+\infty}(\Omega)\right), \\
\left(u^{0}, v^{0}\right) \in\left(W^{1, p}(\Omega) \times W^{1, q}(\Omega)\right) \cap\left(L^{+\infty}(\Omega) \times L^{+\infty}(\Omega)\right), \\
-\Delta_{p} u_{0}-f\left(x, u_{0}, v\right) \leq 0 \leq-\Delta_{p} u^{0}-f\left(x, u^{0}, v\right) \quad \text { in } \Omega, \forall v \in\left[v_{0}, v^{0}\right], \\
-\Delta_{q} v_{0}-f\left(x, u, v_{0}\right) \leq 0 \leq-\Delta_{q} v^{0}-f\left(x, u, v^{0}\right) \quad \text { in } \Omega, \forall u \in\left[u_{0}, u^{0}\right], \\
u_{0} \leq u^{0}, \quad v_{0} \leq v^{0} \quad \text { in } \Omega, \quad u_{0} \leq 0 \leq u^{0}, \quad v_{0} \leq 0 \leq v^{0} \quad \text { on } \partial \Omega .
\end{gathered}
$$

Similar definitions can be found in Diaz and Hernández [7], and Diaz and Herrero [8]. For all $M>0$, we note that

$$
\begin{array}{cl}
\hat{f}(u, v)=f(u, v)+M, & \hat{g}(u, v)=g(u, v)+M, \\
\hat{F}(u, v)=F(u, v)+M u, & \hat{G}(u, v)=G(u, v)+M v .
\end{array}
$$

Notice that if $F$ and $G$ satisfy the assumption (2.1) of $\left(\mathrm{H}_{2}\right)$, then the same holds for $\hat{F}$ and $\hat{G}$.

Proposition 3.2 [6]. Under hypothesis (2.1) of $\left(H_{2}\right)$, there exist two sequences $d_{k}$ and $d_{k}^{\prime}$ such that

(a) $m_{k}^{1 / p} \geq d_{k} \geq 0$, for all $k \in \mathbb{N}$ and

$$
\int_{0}^{d_{k}} \frac{d s}{\sqrt[p]{p \hat{F}\left(d_{k}, m_{k}^{1 / q}\right)-p \hat{F}\left(s, m_{k}^{1 / q}\right)}}>\int_{0}^{1} \frac{d s}{\sqrt[p]{1-s^{p}}}\left[\mu_{p}\right]^{-1 / p}
$$

(b) $m_{k}^{1 / p} \geq d_{k}^{\prime} \geq 0$, for all $k \in N$ and

$$
\int_{0}^{d_{k}^{\prime}} \frac{d t}{\sqrt[q]{q \hat{G}\left(m_{k}^{1 / p}, d_{k}^{\prime}\right)-q \hat{G}\left(m_{k}^{1 / p}, t\right)}}>\int_{0}^{1} \frac{d t}{\sqrt[q]{1-t^{q}}}\left[\mu_{q}\right]^{-1 / q} .
$$

REMARK 3.3. We have

$$
\sqrt[p]{p-1} \int_{0}^{1} \frac{d s}{\sqrt[p]{1-s^{p}}}\left[\mu_{p}\right]^{-1 / p}=\sqrt[q]{q-1} \int_{0}^{1} \frac{d t}{\sqrt[q]{1-t^{q}}}\left[\mu_{q}\right]^{-1 / q}=\frac{b-a}{2}
$$

Proof of Proposition 3.2. We only prove (a); the proof of (b) is similar.

(1) From (2.1) of hypothesis $\left(\mathrm{H}_{2}\right)$, there exists some $\mu>0$ such that

$$
\lim _{k \rightarrow+\infty} \frac{p \hat{F}\left(m_{k}^{1 / p}, m_{k}^{1 / q}\right)}{m_{k}}<\mu<\mu_{p}
$$

then

$$
\lim _{k \rightarrow+\infty} \mu m_{k}-p \hat{F}\left(m_{k}^{1 / p}, m_{k}^{1 / q}\right)=+\infty
$$


(2) We consider the functions $\left[H\left(\cdot, m_{k}\right)\right]_{k}$, where

$$
H\left(s, m_{k}\right)=\mu s-p \hat{F}\left(s^{1 / p}, m_{k}^{1 / q}\right) .
$$

For all $k>0$, we have

$$
\begin{gathered}
H\left(0, m_{k}\right)=-p \hat{F}\left(0, m_{k}^{1 / q}\right)=0, \\
H\left(m_{k}, m_{k}\right)=\mu m_{k}-p \hat{F}\left(m_{k}^{1 / p}, m_{k}^{1 / q}\right)>0 .
\end{gathered}
$$

Then for all $k \in \mathbb{N}$ there exists $d_{k}>0$ such that $d_{k}^{p} \leq m_{k}$ and for all $s \in\left[0, d_{k}^{p}\right]$, we have

$$
H\left(s, m_{k}\right) \leq H\left(d_{k}^{p}, m_{k}\right)
$$

that is,

$$
\mu s-p \hat{F}\left(s^{1 / p}, m_{k}^{1 / q}\right) \leq \mu d_{k}^{p}-p F\left(d_{k}, m_{k}^{1 / q}\right),
$$

then

$$
p \hat{F}\left(d_{k}, m_{k}^{1 / q}\right)-p \hat{F}\left(s^{1 / p}, m_{k}^{1 / q}\right) \leq \mu\left(d_{k}^{p}-s\right) .
$$

Let $s=\omega^{p}$, where $\omega \in\left[0, d_{k}\right] \subset\left[0, m_{k}^{1 / p}\right]$. We obtain

$$
p \hat{F}\left(d_{k}, m_{k}^{1 / q}\right)-p \hat{F}\left(\omega, m_{k}^{1 / q}\right) \leq \mu\left(d_{k}^{p}-\omega^{p}\right),
$$

that is,

$$
\frac{1}{\sqrt[p]{d_{k}^{p}-\omega^{p}}}[\mu]^{-1 / p} \leq \frac{1}{\sqrt[p]{p \hat{F}\left(d_{k}, m_{k}^{1 / q}\right)-p \hat{F}\left(\omega, m_{k}^{1 / q}\right)}} .
$$

Then integrating on $\left[0, d_{k}\right]$ we obtain

$$
\int_{0}^{1} \frac{d \omega}{\sqrt[p]{1-\omega^{p}}}[\mu]^{-1 / p} \leq \int_{0}^{d_{k}} \frac{d \omega}{\sqrt[p]{p \hat{F}\left(d_{k}, m_{k}^{1 / q}\right)-p \hat{F}\left(\omega, m_{k}^{1 / q}\right)}}
$$

This proves (a).

3.1. Construction of supersolution $\left(u^{0}, v^{0}\right)$. In the following step we suppose that for all $k \in \mathbb{N}$ and for all $s \in\left[0, m_{k}^{1 / p}\right]$

$$
f\left(s, m_{k}^{1 / q}\right)+M \geq 0 .
$$

Denote by $\left(\hat{f}_{k}\right)_{k}$ the sequence of functions defined by

$$
\hat{f}_{k}(s)= \begin{cases}f\left(m_{k}^{1 / p}, m_{k}^{1 / q}\right)+M & \text { for } s \text { in }\left[m_{k}^{1 / p},+\infty[\right. \\ f\left(s, m_{k}^{1 / q}\right)+M & \text { for } s \text { in }\left[0, m_{k}^{1 / p}\right], \\ f\left(0, m_{k}^{1 / q}\right)+M & \text { for } s \text { in }]-\infty, 0] .\end{cases}
$$


For all $k \in \mathbb{N}$, we associate to the function $\hat{f}_{k}$, the following problem:

$$
-\left(\left|u^{\prime}\right|^{p-2} u^{\prime}\right)^{\prime}(t)=\hat{f}_{k}(u(t)), \quad u(t) \geq 0 \text { for } t \text { in }[a, b] .
$$

For all $k \in \mathbb{N}$, we define the nonlinear operator $T_{k}$ such that

$$
T_{k}: C([a, b]) \rightarrow C([a, b])
$$

in the following way:

$$
T_{k}(u)(t)=d_{k}-\int_{a}^{t}\left[\int_{a}^{r} \hat{f}_{k}(u(s)) d s\right]^{1 /(p-1)} d r .
$$

Since $\hat{f}_{k}$ is a nonnegative function, the operator $T_{k}$ is well defined.

LEMMA 3.4. For all $k \geq 0$,

(i) the operator $T_{k}$ is completely continuous,

(ii) there exists a fixed point for $T_{k}$.

Proof. Let $k \in \mathbb{N}$,

(1) the continuity is immediate,

(2) let $\left(u_{n}\right)_{n}$ be a bounded sequence in $C([a, b])$ such that the sequence $\left(T_{k}\left(u_{n}\right)\right)_{n}$ is also bounded in $C([a, b])$.

By the continuity of the function $\hat{f}_{k}$, there exists some constant $C_{k}$ such that

$$
t^{\prime} \in[a, b], \quad \forall t
$$

for all $n \in \mathbb{N}$ we have

$$
\left|T_{k}\left(u_{n}\right)(t)-T_{k}\left(u_{n}\right)\left(t^{\prime}\right)\right| \leq C_{k}\left|t-t^{\prime}\right|
$$

So $\left(T_{k}\left(u_{n}\right)\right)_{n}$ is uniformly equicontinuous and by Ascoli theorem the sequence $\left(T_{k}\left(u_{n}\right)\right)_{n}$ is relatively compact in $C([a, b])$.

(3) Using the Leray-Schauder theorem we deduce that $T_{k}$ has a fixed point $u_{k} \in$ $C([a, b])$, that is, $T_{k}\left(u_{k}\right)=u_{k}$.

REMARK 3.5. By definition of the operator $T_{k}$, we have

(i) $-\left|u_{k}^{\prime}\right|^{p-2} u_{k}^{\prime}(t)=\int_{a}^{t} \hat{f}_{k}\left(u_{k}(s)\right) d s$,

(ii) $u_{k}^{\prime}(a)=0$,

(iii) $u_{k}(a)=d_{k}$.

Since $\hat{f}_{k}$ is a nonnegative function we have

(iv) $u_{k}^{\prime}(t) \leq 0$ for $t$ in $[a, b]$.

That is, $u_{k}$ is a nonincreasing function on $[a, b]$.

LEMMA 3.6. From (2.1), choose $\left(d_{k}\right)_{k}$ such that

$$
u_{k}(t) \geq 0 \quad \text { in }\left[a, \frac{a+b}{2}\right] \forall k \in \mathbb{N},
$$

where $u_{k}$ is the fixed point of the operator $T_{k}$. 
Proof. Let $\left(d_{k}\right)_{k}$ be some sequence such that $d_{k} \in\left[0, m_{k}^{1 / p}\right]$ for all $k \in \mathbb{N}$.

We denote by $t_{k}$ a real number such that $u_{k}\left(t_{k}\right)=0$ and $u_{k}(t) \geq 0$ on $\left[a, t_{k}\right]$.

Then, from Remark 3.5, since $u_{k}$ is a nonincreasing function and $d_{k} \in\left[0, m_{k}^{1 / p}\right]$, we have

$$
m_{k}^{1 / p} \geq u_{k} \geq 0 \quad \forall t \in\left[a, t_{k}\right]
$$

Consequently, for all $t \in\left[a, t_{k}\right]$ we have

$$
-\left(\left|u_{k}^{\prime}\right|^{p-2} u_{k}^{\prime}\right)^{\prime}(t)=\hat{f}_{k}\left(u_{k}(t)\right)=f\left(u_{k}(t), m_{k}^{1 / q}\right)+M .
$$

Multiplying (3.25) by $u_{k}^{\prime}$ we obtain

$$
\frac{p-1}{p}\left(-\left|u_{k}^{\prime}(t)\right|^{p}\right)^{\prime}=\frac{d}{d t}\left(\hat{F}\left(u_{k}(t), m_{k}^{1 / q}\right)\right),
$$

where

$$
\hat{F}\left(u, m_{k}^{1 / q}\right)=F(u, v)+M u
$$

Integrating (3.26) on $[a, t] \subset\left[a, t_{k}\right]$, we obtain

$$
-\sqrt[p]{p-1} u_{k}^{\prime}(t)=\sqrt[p]{p \hat{F}\left(d_{k}, m_{k}^{1 / q}\right)-p \hat{F}\left(u_{k}(t), m_{k}^{1 / q}\right)} .
$$

Integrating (3.28) again on $\left[a, t_{k}\right]$ we deduce that

$$
\sqrt[p]{p-1} \int_{a}^{t_{k}} \frac{-u_{k}^{\prime}(t)}{\sqrt[p]{p \hat{F}\left(d_{k}, m_{k}^{1 / q}\right)-p \hat{F}\left(u_{k}(t), m_{k}^{1 / q}\right)}} d t \leq t_{k}-a
$$

Then, we obtain

$$
\sqrt[p]{p-1} \int_{0}^{d_{k}} \frac{1}{\sqrt[p]{p \hat{F}\left(d_{k}, m_{k}^{1 / q}\right)-p \hat{F}\left(s, m_{k}^{1 / q}\right)}} d s \leq t_{k}-a
$$

It follows from Proposition 3.2 and Remark 3.3 that one can choose the sequence $\left(d_{k}\right)$ such that for all $k \geq k_{0}$ we have

$$
\frac{b-a}{2}<\sqrt[p]{p-1} \int_{0}^{d_{k}} \frac{1}{\sqrt[p]{p \hat{F}\left(d_{k}, m_{k}^{1 / q}\right)-p \hat{F}\left(s, m_{k}^{1 / q}\right)}} d s .
$$

Consequently, from (3.30) and (3.31), we obtain that for all $k \geq k_{0}$, there exists $t_{k}$ satisfying $t_{k}>(b+a) / 2$.

Proposition 3.7. Suppose that the sequence $\left(m_{k}\right)_{k}$ satisfies (2.1), and that for all $k>0$ we have

$$
\inf _{s \in\left[0, m_{k}^{1 / p}\right]} f\left(s, m_{k}^{1 / q}\right)+M \geq 0 .
$$


Then, there exists some number $k_{0} \in \mathbb{N}$ such that for all $k \geq k_{0}$ the problem

$$
\begin{gathered}
-\left(\left|u^{\prime}\right|^{p-2} u^{\prime}\right)^{\prime}=f\left(u, m_{k}^{1 / q}\right)+M \quad \text { in }(a, b), \\
u \geq 0 \quad \text { on }[a, b],
\end{gathered}
$$

has a solution $\hat{u}_{k}$ satisfying $\hat{u}_{k} \in C^{1}([a, b]),\left(\left|\hat{u}_{k}^{\prime}\right|^{p-2} \hat{u}_{k}^{\prime}\right)^{\prime} \in C([a, b])$ and $m_{k}^{1 / p} \geq \hat{u}_{k} \geq 0$ for all $k \geq k_{0}$.

Proof. Let $\left(u_{k}\right)_{k}$ be the sequence defined in Lemma 3.6. This sequence satisfies that for all $k \geq k_{0}$,

$$
\begin{gathered}
u_{k} \in C^{1}\left(\left[a, \frac{a+b}{2}\right]\right), \quad\left(\left|u_{k}^{\prime}\right|^{p-2} u_{k}^{\prime}\right)^{\prime} \in C\left(\left[a, \frac{a+b}{2}\right]\right), \\
-\left(\left|u_{k}^{\prime}\right|^{p-2} u_{k}^{\prime}\right)^{\prime}(t)=f\left(u_{k}(t), m_{k}^{1 / q}\right)+M \quad \text { in }\left[a, \frac{a+b}{2}\right], \\
m_{k}^{1 / p} \geq \hat{u}_{k} \geq 0 \quad \text { in }\left[a, \frac{a+b}{2}\right], \\
u_{k}^{\prime}(a)=0 .
\end{gathered}
$$

We denote by $\hat{u}_{k}$ the following function:

$$
\hat{u}_{k}(t)= \begin{cases}u_{k}\left(\frac{3 a+b}{2}-t\right) & \text { if } t \in\left[a, \frac{a+b}{2}\right], \\ u_{k}\left(t-\frac{a+b}{2}\right) & \text { if } t \in\left[\frac{a+b}{2}, b\right] .\end{cases}
$$

Then, from (3.34), it is easy to see that

$$
\begin{gathered}
\forall k \geq k_{0}, \quad \hat{u}_{k} \in C^{1}([a, b]), \quad\left(\left|\hat{u}_{k}^{\prime}\right|^{p-2} \hat{u}_{k}^{\prime}\right)^{\prime} \in C([a, b]), \\
-\left(\left|\hat{u}_{k}^{\prime}\right|^{p-2} \hat{u}_{k}^{\prime}\right)^{\prime}(t)=f\left(\hat{u}_{k}(t), m_{k}^{1 / q}\right)+M \quad \text { in }[a, b], \\
m_{k}^{1 / p} \geq \hat{u}_{k} \geq 0 \quad \text { in }[a, b] .
\end{gathered}
$$

Then the conclusion holds.

Proposition 3.8. Let $M>0$. From (2.1), there exist some $m>0$ and $\left(\hat{u}_{m}, \hat{v}_{m}\right) \in$ $\left(C^{1}([a, b])\right)^{2}$ such that

$$
\begin{aligned}
& \left(\left(\left|\hat{u}_{m}^{\prime}\right|^{p-2} \hat{u}_{m}^{\prime}\right)^{\prime},\left(\left|\hat{v}_{m}^{\prime}\right|^{p-2} \hat{v}_{m}^{\prime}\right)^{\prime}\right) \in(C[a, b])^{2}, \\
& -\left(\left|\hat{u}_{m}^{\prime}\right|^{p-2} \hat{u}_{m}^{\prime}\right)^{\prime} \geq f\left(\hat{u}_{m}, m^{1 / q}\right)+M \quad \text { in }(a, b), \\
& -\left(\left|\hat{v}_{m}^{\prime}\right|^{q-2} \hat{v}_{m}^{\prime}\right)^{\prime} \geq g\left(m^{1 / p}, \hat{v}_{m}\right)+M \quad \text { in }(a, b), \\
& m^{1 / p} \geq \hat{u}_{m} \geq 0, \quad m^{1 / q} \geq \hat{v}_{m} \geq 0 \quad \text { on }[a, b] .
\end{aligned}
$$


Proof. We study three cases.

CASE 1. We suppose that for all $k \in \mathbb{N}$ we have

$$
\inf _{s \in g\left[0, m_{k}^{1 / p}\right]} f\left(s, m_{k}^{1 / q}\right)+M<0, \quad \inf _{t \in\left[0, m_{k}^{1 / q}\right]} g\left(m_{k}^{1 / p}, t\right)+M<0 .
$$

Then for all $k \in \mathbb{N}$, there exist $s_{m_{k}} \in\left[0, m_{k}^{1 / p}\right]$ and $t_{m_{k}} \in\left[0, m_{k}^{1 / q}\right]$ satisfying

$$
f\left(s_{m_{k}}, m_{k}^{1 / q}\right)+M<0, \quad g\left(m_{k}^{1 / p}, t_{m_{k}}\right)+M<0 .
$$

Consequently, for $m=m_{k}$, the couple $\left(\hat{u}_{m}, \hat{v}_{m}\right)=\left(s_{m_{k}}, t_{m_{k}}\right)$ satisfies the result.

CASE 2. Assume that for all $k \in \mathbb{N}$ we have,

$$
\begin{gathered}
\inf _{s \in\left[0, m_{k}^{1 / p}\right]} f\left(s, m_{k}^{1 / q}\right)+M \geq 0, \\
\inf _{t \in\left[0, m_{k}^{1 / q}\right]} g\left(m_{k}^{1 / p}, t\right)+M<0 .
\end{gathered}
$$

(a) From (3.41) and Proposition 3.7 there exist some $k_{0} \in \mathbb{N}$ and some sequence $\left(\hat{u}_{k}\right)_{k}$ such that, for all $k \geq k_{0}$, we have

$$
\begin{gathered}
\hat{u}_{k} \in C^{1}([a, b]), \quad\left(\left|\hat{u}_{k}^{\prime}\right|^{p-2} \hat{u}_{k}^{\prime}\right)^{\prime} \in C([a, b]), \\
-\left(\left|\hat{u}_{k}^{\prime}\right|^{p-2} \hat{u}_{k}^{\prime}\right)^{\prime} \geq f\left(\hat{u}_{k}, m_{k}^{1 / q}\right)+M \quad \text { in }(a, b), \\
m_{k}^{1 / p} \geq \hat{u}_{k} \geq 0 \quad \text { in }[a, b] .
\end{gathered}
$$

(b) From (3.42), there exists a sequence $\left(t_{m_{k}}\right)_{k}$ such that

$$
m_{k}^{1 / p} \geq t_{m_{k}} \geq 0, \quad g\left(m_{k}^{1 / p}, t_{m_{k}}\right)+M<0 \quad \forall k \geq k_{0} .
$$

Consequently, for $m=m_{k}$ with $k>k_{0}$, the pair $\left(\hat{u}_{m_{k}}, t_{m_{k}}\right)$ satisfies the result.

CASE 3. Assume that for all $k \in \mathbb{N}$, we have

$$
\inf _{s \in\left[0, m_{k}^{1 / p}\right]} f\left(s, m_{k}^{1 / q}\right)+M \geq 0, \quad \inf _{t \in\left[0, m_{k}^{1 / q}\right]} g\left(m_{k}^{1 / p}, t\right)+M \geq 0 .
$$

Then, from Proposition 3.7, for all $k \geq k_{0}$, there exists $\left(\hat{u}_{k}, \hat{v}_{k}\right) \in\left(C^{1}([a, b])\right)^{2}$ such that

$$
\begin{gathered}
\left(\left(\left|\hat{u}_{k}^{\prime}\right|^{p-2} \hat{u}_{k}^{\prime}\right)^{\prime},\left(\left|\hat{v}_{k}^{\prime}\right|^{p-2} \hat{v}_{k}^{\prime}\right)^{\prime}\right) \in(C[a, b])^{2}, \\
-\left(\left|\hat{u}_{k}^{\prime}\right|^{p-2} \hat{u}_{k}^{\prime}\right)^{\prime} \geq f\left(\hat{u}_{k}, m_{k}^{1 / q}\right)+M \quad \text { in }(a, b), \\
-\left(\left|\hat{v}_{k}^{\prime}\right|^{p-2} \hat{v}_{k}^{\prime}\right)^{\prime} \geq g\left(m_{k}^{1 / p}, \hat{v}_{k}\right)+M \quad \text { in }(a, b), \\
m_{k}^{1 / p} \geq \hat{u}_{k} \geq 0, \quad m_{k}^{1 / q} \geq \hat{v}_{k} \geq 0 \quad \text { on }[a, b] .
\end{gathered}
$$

This proves the results.

Now, for problem (1.1), we consider a smooth bounded domain $\Omega$ in $\mathbb{R}^{N}$, and we have the following result. 
Proposition 3.9. Under hypotheses $\left(H_{1}\right)$ and (2.1) of $\left(H_{2}\right)$, problem (1.1) has a nonnegative supersolution $\left(u^{0}, v^{0}\right)$ in $W^{1, p}(\Omega) \times W^{1, q}(\Omega)$.

Proof. Let $M \geq\left\|h_{1}\right\|_{\infty}+\left\|h_{2}\right\|_{\infty} \cdot P=\prod\left[a_{i}, b_{i}\right]$ is a cube containing $\Omega$ and

$$
b-a=\inf _{1 \leq i \leq N} b_{i}-a_{i}=b_{1}-a_{1} .
$$

From (2.1) of hypothesis $\left(\mathrm{H}_{2}\right)$ and Proposition 3.8, there exist $m>0$ and $\left(\hat{u}_{m}, \hat{v}_{m}\right) \in$ $\left(C^{1}([a, b])\right)^{2}$ such that

$$
\left(\left(\left|\hat{u}_{m}^{\prime}\right|^{p-2} \hat{u}_{m}^{\prime}\right)^{\prime},\left(\left|\hat{v}_{m}^{\prime}\right|^{p-2} \hat{v}_{m}^{\prime}\right)^{\prime}\right) \in(C[a, b])^{2},
$$

and $\left(\hat{u}_{m}, \hat{v}_{m}\right)$ satisfies

$$
\begin{gathered}
-\left(\left|\hat{u}_{m}^{\prime}\right|^{p-2} \hat{u}_{m}^{\prime}\right)^{\prime} \geq f\left(\hat{u}_{m}, m^{1 / q}\right)+M \quad \text { in }(a, b), \\
-\left(\left|\hat{v}_{m}^{\prime}\right|^{q-2} \hat{v}_{m}^{\prime}\right)^{\prime} \geq g\left(m^{1 / p}, \hat{v}_{m}\right)+M \quad \text { in }(a, b), \\
m^{1 / p} \geq \hat{u}_{m} \geq 0, \quad m^{1 / q} \geq \hat{v}_{m} \geq 0 \quad \text { on }[a, b] .
\end{gathered}
$$

We denote by $u^{0}$ and $v^{0}$ the functions such that for all $x \in \Omega$ with $x=\left(x_{1}, x_{2}, \ldots, x_{N}\right)$

$$
u^{0}(x)=\hat{u}_{m}\left(x_{1}\right), \quad v^{0}(x)=\hat{v}_{m}\left(x_{1}\right)
$$

where $\left(u^{0}, v^{0}\right)$ is clearly in $W^{1, p}(\Omega) \times W^{1, q}(\Omega)$, moreover by hypothesis $\left(\mathrm{H}_{1}\right)$, we easily obtain

$$
\begin{gathered}
-\Delta_{p} u^{0} \geq f\left(u^{0}, v\right)+h_{1} \text { for } v \leq v^{0} \text { a.e. on } \Omega, \\
-\Delta_{q} v^{0} \geq g\left(u, v^{0}\right)+h_{2} \text { for } u \leq u^{0} \text { a.e. on } \Omega, \\
u^{0} \geq 0, \quad v^{0} \geq 0 \quad \text { on } \Omega .
\end{gathered}
$$

Then the result follows.

3.2. Construction of a subsolution $\left(u_{0}, v_{0}\right)$. Similar to the construction of a supersolution we can prove the following result.

Proposition 3.10. Under hypotheses $\left(H_{1}\right)$ and (2.2) of $\left(H_{2}\right)$, problem (1.1) has a subsolution $\left(u_{0}, v_{0}\right)$ in $W^{1, p}(\Omega) \times W^{1, q}(\Omega)$.

4. Proof of Theorem 2.1. We proceed in the following steps.

(i) From Propositions 3.9 and 3.10, there exists a pair $\left[\left(u_{0}, v_{0}\right) ;\left(u^{0}, v^{0}\right)\right]$ of subsupersolution of problem (1.1).

(ii) Construction of an invariant set. In order to apply Schauder's fixed point theorem, we introduce the set $K=\left[u_{0}, u^{0}\right] \times\left[v_{0}, v^{0}\right]$. Next we define the following nonlinear operator $T$ : for all $\left(u_{1}, v_{1}\right) \in W^{1, p}(\Omega) \times W^{1, q}(\Omega)$, we associate $\left(u_{2}, v_{2}\right)=$ $T\left(\left(u_{1}, v_{1}\right)\right)$, where $\left(u_{2}, v_{2}\right)$ is the solution of the system

$$
\begin{gathered}
-\Delta_{p} u=\hat{f}\left(x, u, v_{1}\right), \quad-\Delta_{q} v=\hat{g}\left(x, u_{1}, v\right) \quad \text { in } \Omega, \\
u=0, \quad v=0 \quad \text { on } \partial \Omega,
\end{gathered}
$$

where

$$
\hat{f}(x, u, v)=f(U, V)+h_{1}(x), \quad \hat{g}(x, u, v)=g(U, V)+h_{2}(x),
$$


with

$$
\begin{aligned}
& U(x)=u(x)+\left(u_{0}-u\right)_{+}-\left(u-u^{0}\right)_{+}, \\
& V(x)=v(x)+\left(v_{0}-v\right)_{+}-\left(v-v^{0}\right)_{+} .
\end{aligned}
$$

The functions $\hat{f}$ and $\hat{g}$ are bounded, so the operator $T$ is well defined. Furthermore, $K$ is an invariant set for $T$. Let $\left(u_{1}, v_{1}\right) \in K$ and $\left(u_{2}, v_{2}\right)=T\left(\left(u_{1}, v_{1}\right)\right)$.

We show, for example, that $u_{2} \leq u^{0}$. From (3.51), (4.1), and (4.2) we have

$$
\begin{aligned}
0 & \geq-\Delta_{p} u_{2}-\hat{f}\left(x, u_{2}, v_{1}\right) \geq-\Delta_{p} u_{2}-f\left(U_{2}, V_{1}\right)-h_{1}(x) \\
& \geq\left[-\Delta_{p} u_{2}+\Delta_{p} u^{0}\right]+\left[f\left(u^{0}, v_{1}\right)-f\left(U_{2}, V_{1}\right)\right],
\end{aligned}
$$

multiplying (4.4) by $\left(u_{2}-u^{0}\right)_{+}$and integrating over $\Omega$, we obtain

$$
\begin{aligned}
0 \geq & \int_{\Omega}\left[\left|\nabla u_{2}\right|^{p-2} \nabla u_{2}-\left|\nabla u^{0}\right|^{p-2} \nabla u^{0}\right] \nabla\left(u_{2}-u^{0}\right)_{+} d x \\
& +\int_{\Omega}\left[f\left(u^{0}, v_{1}\right)-f\left(U_{2}, V_{1}\right)\right]\left(u_{2}-u^{0}\right)_{+} d x .
\end{aligned}
$$

Since $v_{1} \in\left[v_{0}, v^{0}\right]$, we have $V_{1}=v_{1}$, where $V_{1}$ is associated with $v_{1}$ as in (4.3).

Denote by $\Omega_{+}$the set

$$
\Omega_{+}=\left\{x \in \Omega ; u_{2}-u^{0}>0\right\} .
$$

We have $U_{2}=u^{0}$ in $\Omega_{+}$. Then

$$
\begin{aligned}
\int_{\Omega}[ & \left.f\left(u^{0}, v_{1}\right)-f\left(U_{2}, V_{1}\right)\right]\left(u_{2}-u^{0}\right)_{+} d x \\
& =\int_{\Omega}\left[f\left(u^{0}, v_{1}\right)-f\left(u_{0}, v_{1}\right)\right]\left(u_{2}-u^{0}\right)_{+} d x=0 .
\end{aligned}
$$

By the monotonicity of $-\Delta_{p}$ in $L^{p}(\Omega)$, we get that $0 \geq\left\|\left(u_{2}-u^{0}\right)_{+}\right\|_{L^{p}(\Omega)}$.

Thus $u_{2} \leq u^{0}$ on $\Omega$ and similarly $v_{2} \leq v^{0}$ on $\Omega$. So that the property, $T(K) \subset K$, holds.

(iii) The operator $T$ is completely continuous.

(a) We prove that $T$ is compact; let $\left(u_{1}^{j}, v_{1}^{j}\right)_{j}$ be a bounded sequence in $L^{p}(\Omega) \times$ $L^{q}(\Omega)$. Let $\left(u_{2}^{j}, v_{2}^{j}\right)=T\left(\left(u_{1}^{j}, v_{1}^{j}\right)\right)$, so multiplying $(4.1)$ by $u_{2}^{j}$, we obtain

$$
\int_{\Omega}\left|\nabla u_{2}^{j}\right|^{p} d x=\int_{\Omega} \hat{f}\left(x, u_{2}^{j}, v_{1}^{j}\right) u_{2}^{j} d x \leq C\left[\int_{\Omega}\left|u_{2}^{j}\right|^{p} d x\right]^{1 / p} .
$$

Therefore, $\left(u_{2}^{j}\right)_{j}$ is bounded in $W^{1, p}(\Omega)$ and it possesses a convergent subsequence in $L^{p}(\Omega)$. Analogously for $\left(v_{2}^{j}\right)_{j}$ in $L^{q}(\Omega)$.

(b) Now we prove the continuity of the operator $T$; from the continuity of the functions $f$ and $g$ associated at the bounded functions $\hat{f}, \hat{g}$, and by the dominated convergence theorem, we deduce easily the continuity of the operator $T$.

Since $K$ is a convex, bounded, and closed subset, we apply Schauder's fixed point theorem and we obtain the existence of a fixed point for $T$ which gives the existence of one solution of (1.1). 
ACKNOWLEDGMENTS. A part of this work was made when the author was visiting the Laboratory MIP of the University of Toulouse-3.

The author also thanks Professor François de Thélin for useful discussions.

\section{REFERENCES}

[1] A. Anane and O. Chakrone, Bème cycle, thesis, Mohammed I University, Oujda, Morocco, 1996.

[2] C. Baoyao, Nonexistence results and existence theorems of positive solutions of Dirichlet problems for a class of semilinear elliptic systems of second order, Acta Math. Sci. (English Ed.) 7 (1987), no. 3, 299-309.

[3] L. Boccardo, J. Fleckinger, and F. de Thélin, Elliptic systems with various growth, Reaction Diffusion Systems (Trieste, 1995), Lecture Notes in Pure and Appl. Math., 194, Dekker, New York, 1998, pp. 59-66.

[4] H. Brézis and E. H. Lieb, Minimum action solutions of some vector field equations, Comm. Math. Phys. 96 (1984), no. 1, 97-113.

[5] P. Clément, R. Manásevich, and E. Mitidieri, Positive solutions for a quasilinear system via blow up, Comm. Partial Differential Equations 18 (1993), no. 12, 2071-2106.

[6] D. G. de Figueiredo and J.-P. Gossez, Nonresonance below the first eigenvalue for a semilinear elliptic problem, Math. Ann. 281 (1988), no. 4, 589-610.

[7] J. I. Diaz and J. Hernández, On the existence of a free boundary for a class of reactiondiffusion systems, SIAM J. Math. Anal. 15 (1984), no. 4, 670-685.

[8] J. I. Diaz and M. A. Herrero, Estimates on the support of the solutions of some nonlinear elliptic and parabolic problems, Proc. Roy. Soc. Edinburgh Sect. A 89 (1981), no. 3-4, 249-258.

[9] P. Felmer, R. F. Manásevich, and F. de Thélin, Existence and uniqueness of positive solutions for certain quasilinear elliptic systems, Comm. Partial Differential Equations 17 (1992), no. 11-12, 2013-2029.

[10] M. L. C. Fernandes, P. Omari, and F. Zanolin, On the solvability of a semilinear two-point BVP around the first eigenvalue, Differential Integral Equations 2 (1989), no. 1, 63-79.

[11] A. Fonda, J.-P. Gossez, and F. Zanolin, On a nonresonance condition for a semilinear elliptic problem, Differential Integral Equations 4 (1991), no. 5, 945-951.

[12] J.-P. Gossez and A. El Hachimi, On a nonresonance condition near the first eigenvalue for a quasilinear elliptic problem, thesis, Univ. Catholique de Louvain, France, 1994.

[13] J. Vélin and F. de Thélin, Existence and nonexistence of nontrivial solutions for some nonlinear elliptic systems, Rev. Mat. Univ. Complut. Madrid 6 (1993), no. 1, 153-194.

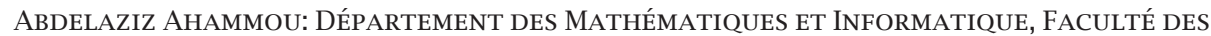
Sciences, Université Chouaib DoukKali, BP 20, 24000 El JADIDA, Morocco

E-mail address: ahammou@ucd.ac.ma 


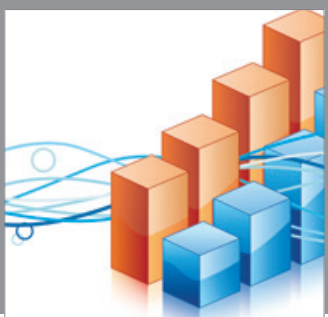

Advances in

Operations Research

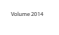

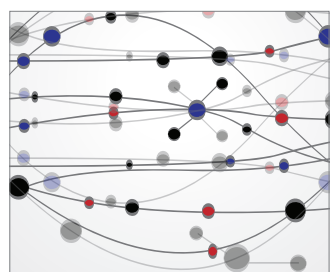

\section{The Scientific} World Journal
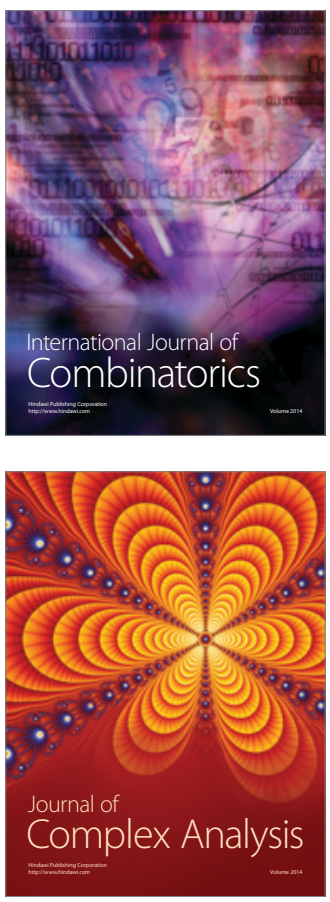

International Journal of

Mathematics and

Mathematical

Sciences
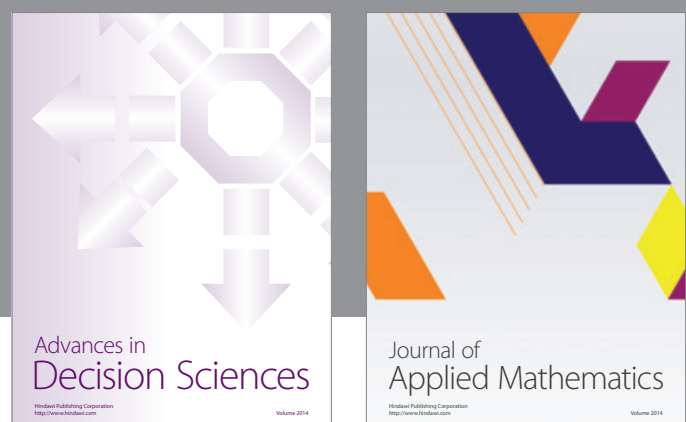

Journal of

Applied Mathematics
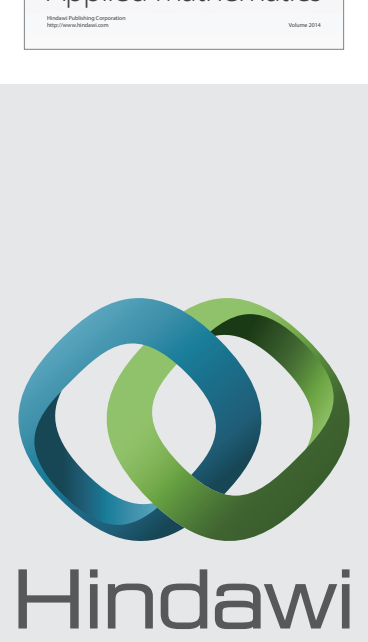

Submit your manuscripts at http://www.hindawi.com
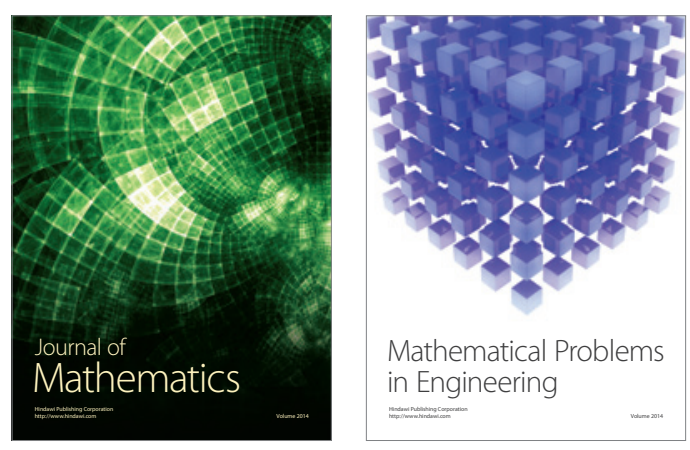

Mathematical Problems in Engineering
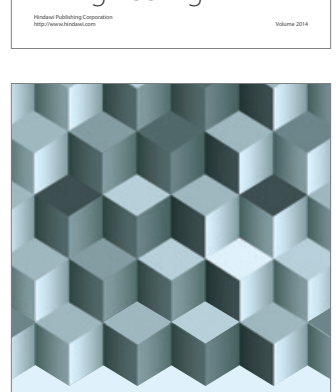

Journal of

Function Spaces
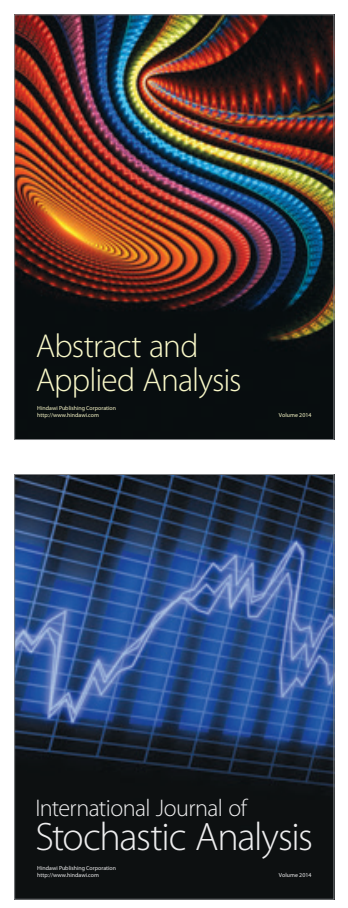

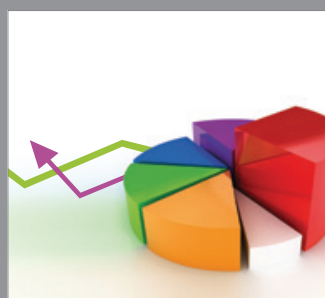

ournal of

Probability and Statistics

Promensencen
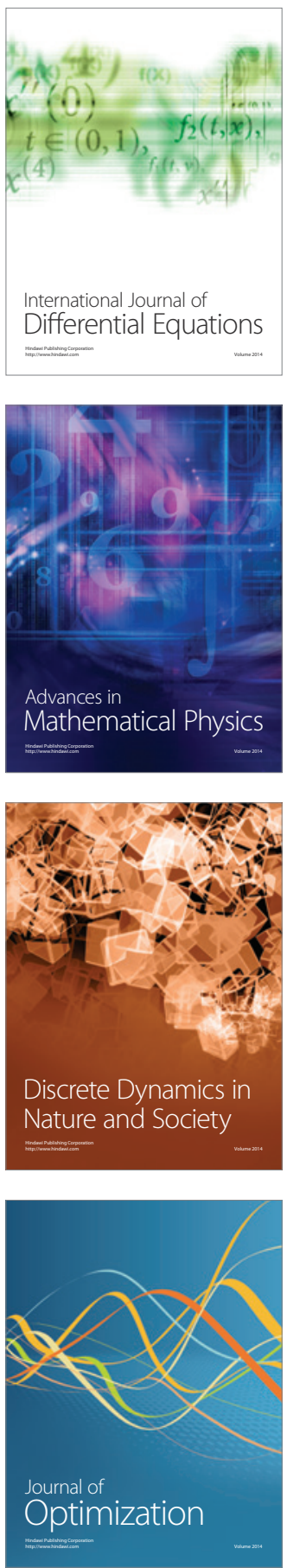